\title{
Anomalous weak values and the violation of a multiple-measurement Leggett-Garg inequality
}

\author{
Alessio Avella, ${ }^{1}$ Fabrizio Piacentini, ${ }^{1}$ Michelangelo Borsarelli, ${ }^{2}$ Marco Barbieri, ${ }^{3}$ Marco Gramegna, ${ }^{1}$ Rudi Lussana, ${ }^{4}$ \\ Federica Villa, ${ }^{4}$ Alberto Tosi, ${ }^{4}$ Ivo Pietro Degiovanni, ${ }^{1}$ and Marco Genovese ${ }^{1}$ \\ ${ }^{1}$ Istituto Nazionale di Ricerca Metrologica, Strada delle Cacce 91, 10135 Torino, Italy \\ ${ }^{2}$ Università degli Studi di Torino, via Pietro Giuria 1, 10125 Torino, Italy \\ ${ }^{3}$ Dipartimento di Scienze, Università degli Studi Roma Tre, Via della Vasca Navale 84, 00146 Rome, Italy \\ ${ }^{4}$ Politecnico di Milano, Dipartimento di Elettronica, Informazione e Bioingegneria, Piazza Leonardo da Vinci 32, 20133 Milano, Italy
}

(Received 1 June 2017; revised manuscript received 2 August 2017; published 20 November 2017)

\begin{abstract}
Quantum mechanics presents peculiar properties that, on the one hand, have been the subject of several theoretical and experimental studies about its very foundations and, on the other hand, provide tools for developing new technologies, the so-called quantum technologies. The nonclassicality pointed out by Leggett-Garg inequalities has represented, with Bell inequalities, one of the most investigated subjects. In this article we study the connection of Leggett-Garg inequalities with a new emerging field of quantum measurement, the weak values in the case of a series of sequential measurements on a single object. In detail, we perform an experimental study of the four-time-correlator Leggett-Garg test, by exploiting single and sequential weak measurements performed on heralded single photons.
\end{abstract}

DOI: 10.1103/PhysRevA.96.052123

\section{INTRODUCTION}

The prominent role of measurement is one of the distinctive features of quantum theory [1]. The impossibility of interpreting the results of a measurement on a quantum system in terms of pre-existing values is the core message of Bell's nonlocality test [2,3], as well as the core message of noncontextuality tests [4]. Such an occurrence has also been recognized by Leggett and Garg [5] in the behavior of macroscopic systems when subject to subsequent measurements. For these objects, it is natural to assume that they will be found in a definite, realistic macrostate (macroscopic realism) and that a measurement, especially when carried out by a microscopic probe, cannot perturb such a macrostate (noninvasive measurability). This original observation by Leggett and Garg has led to a fecund production of theoretical [6-14] and experimental [15-23] work focusing on the inadequacy of such a macrorealistic view [24]; this has also inspired somehow the transposition of Bell's nonlocal argument to the time domain [25-29].

In its simplest form, Leggett and Garg's arrangement considers a macroscopic body undergoing three two-outcome measurements at different times, with the first serving as a preparation. The correlation among the outcomes can be shown not to be in accordance with macrorealistic prescriptions. To date, the violation of the Leggett-Garg inequality has been reported on macroscopic objects, such as transmon qubits [15] and crystals [21], and following refinements have been explored with superconducting flux qubits [22]. The test, however, is also suited to highlight the inadequacy of a realistic view to the description of simpler quantum objects, such as phosphorus impurities [19]. In this case, the focus is on the assessment of the quantum character of the system, being a very significant tool for quantum technologies [14,23,30], rather than on its fundamental value.

The canonic three-measurement arrangement can be generalized in several directions. The simplest extension considers longer sequences $[6,8]$. Increasing the number of measurements leads to stronger departures from the macrorealistic predictions, as has been tested with photons [31] and nuclear spins [18]. A different take considers substituting the measurement in the middle with a weak measurement imparting limited back-action [32]. Different from the standard approach, weak measurements allow for testing the Leggett-Garg inequality in a single apparatus. While shot by shot the measurement delivers only partial information on the observable, it still provides the correct expectation value on a large ensemble [33]. This concept has been introduced in Refs. [34,35] and has been tested on single photons in Ref. [16] and on transmons in Ref. [36]. Remarkably, the number of measurements can also be increased by considering entangled systems, as it has been performed in the semiweak [17] and weak regimes [37].

The experimental scheme for a Leggett-Garg test (LGT) can also be employed for observing so-called postselected values: the value of the second observable is considered only on events chosen according to the outcome of the last measurement. Postselection procedures are expected to be mostly harmless in classical statistics, although the subject is vigorously debated [38-43]; in this context, postselection carried out in the weak-measurement regime can lead to anomalous values, in that they fall outside the range allowed for standard values $[44,45]$. When one allows for such a weak measurement to be performed in a Leggett-Garg test, then a direct connection can be established between the violation of macrorealism and the emergence of anomalous postselected values [16,35,46], like it was demonstrated for quantum contextuality $[47,48]$. Schemes for the measurement of weak values have found technical applications, thanks to their ability to amplify small effects [49-55].

In this article, we present an experiment encompassing these two generalizations at the same time, by demonstrating a sequential multiple-measurement setting carried out in the weak regime. We perform a LGT on the polarization of single photons, estimating noncommuting observables via "weak averages" [56], and draw an explicit link to the emergence of anomalous values. Our experiment confirms the intimate connection between the observation of anomalies in the postselected statistics of quantum measurement as a genuine 
manifestation of the quantum character of the observed object. These anomalies can then be used as practical witnesses of nonclassicality whenever such a test is needed, for instance, in the production of random numbers.

\section{THEORETICAL BACKGROUND}

The simplest LGT one can design involves three measurements, which we label as $I_{A}, I_{B}$, and $I_{C}$; these are two-outcome observables which can take either the value +1 or the value -1 . The inequality can be written as follows [5]:

$$
-3 \leqslant \mathcal{B}_{3}=\left\langle I_{A} I_{B}\right\rangle+\left\langle I_{B} I_{C}\right\rangle-\left\langle I_{A} I_{C}\right\rangle \leqslant 1 .
$$

The measurement of $I_{A}$ can be taken to coincide with the initial preparation in the state $\left|\psi_{A}\right\rangle$ [34]; hence one can assign the fixed value +1 for $I_{A}$ :

$$
-3 \leqslant \mathcal{B}_{3}=\left\langle I_{B}\right\rangle+\left\langle I_{B} I_{C}\right\rangle-\left\langle I_{C}\right\rangle \leqslant 1
$$

The connection with anomalous postselected values of $I_{B}$ is established by considering the two instances $I_{C}=1$ and $I_{C}=-1$ separately, each with the respective occurrence probabilities $p_{C}(1)$ and $p_{C}(-1)$ :

$$
\mathcal{B}_{3}=\left\langle I_{B}\right\rangle+\left[_{+}\left\langle I_{B}\right\rangle-1\right] p_{C}(1)-\left[-\left\langle I_{B}\right\rangle-1\right] p_{C}(-1),
$$

with ${ }_{a}\left\langle I_{B}\right\rangle$ identifying the postselected value of $I_{B}$, conditioned on the outcome $a$ for $I_{C}$. Exploiting the relation

$$
\left\langle I_{B}\right\rangle={ }_{+}\left\langle I_{B}\right\rangle p_{C}(1)+{ }_{-}\left\langle I_{B}\right\rangle p_{C}(-1),
$$

it is possible manipulate Eq. (3) as

$$
\mathcal{B}_{3}=1+2 p_{C}(1)\left(_{+}\left\langle I_{B}\right\rangle-1\right) .
$$

Inserting the condition for the standard values of $\left\langle I_{B}\right\rangle$, one recovers the limits of the Leggett-Garg inequality (LGI).

This connection can be extended to the multiplemeasurement LGT introduced in Ref. [5] that considers four measurements, including state preparation $I_{A}$ :

$$
\left|\mathcal{B}_{4}\right|=\left|\left\langle I_{B}\right\rangle+\left\langle I_{B} I_{C}\right\rangle+\left\langle I_{C} I_{D}\right\rangle-\left\langle I_{D}\right\rangle\right| \leqslant 2 .
$$

The form of this inequality resembles the familiar ClauserHolt-Shimony-Horne test for spacelike separated systems [57]; in that case, two partners alternate four distinct experimental arrangements and verify whether the collected statistics is compatible with a local, realistic theory [25,27]. This can be viewed as a single system interrogated at four different times, including preparation. We can manipulate the fourmeasurement term $\mathcal{B}_{4}$ as we did for its three-measurement counterpart, by distinguishing the two instances for the last measurement $I_{D}$. This is the simplest generalization of the treatment in Ref. [35] and, as we show later, is relevant to our experimental implementation:

$$
\begin{aligned}
\left|\mathcal{B}_{4}\right|= & \mid\left\langle I_{B}\right\rangle+\left\langle I_{B} I_{C}\right\rangle+p_{D}(1)\left[_{+}\left\langle I_{C}\right\rangle-1\right] \\
& -p_{D}(-1)\left[-\left\langle I_{C}\right\rangle-1\right] \mid .
\end{aligned}
$$

We now assume that the postselected values are bound to be found in the same ranges as the standard values: in this case, it is easy to verify that $\left|\mathcal{B}_{4}\right|$ is upper bounded by 2 . The expression (7) reveals a difference with the three-measurement scenario, where a one-to-one correspondence between anomalous weak values and violation of the LGI exists. In our case, violation of the bound for (7) demands a minimal value,

$$
-\left\langle I_{C}\right\rangle \geqslant \frac{3-M}{2 p_{D}(-1)},
$$

where $M=\left\langle I_{B}\right\rangle+\left\langle I_{B} I_{C}\right\rangle+\left\langle I_{C}\right\rangle$, with a similar expression holding for ${ }_{+}\left\langle I_{C}\right\rangle$. We remark that a related behavior is also observed in the monitoring of entangled systems, in which a connection is established between a violation of the LGI and a convex sum of weak values [17]. In the case of sequential measurements as well, this connection persists in a subtler way than in the canonical three-measurement scenario. A more extensive treatment is presented in the Appendix.

\section{EXPERIMENTAL IMPLEMENTATION}

We perform a test of the inequality (7) by exploiting single photons undergoing single and sequential weak measurements of their polarization. Such a test certifies quantum properties of the single photon and uses these to illustrate the link between the LGI and weak values, rather than providing a conclusive proof against macrorealism. In this spirit, we are assuming a certain confidence in our apparatus (Fig. 1); hence we do not address loopholes [37,58].

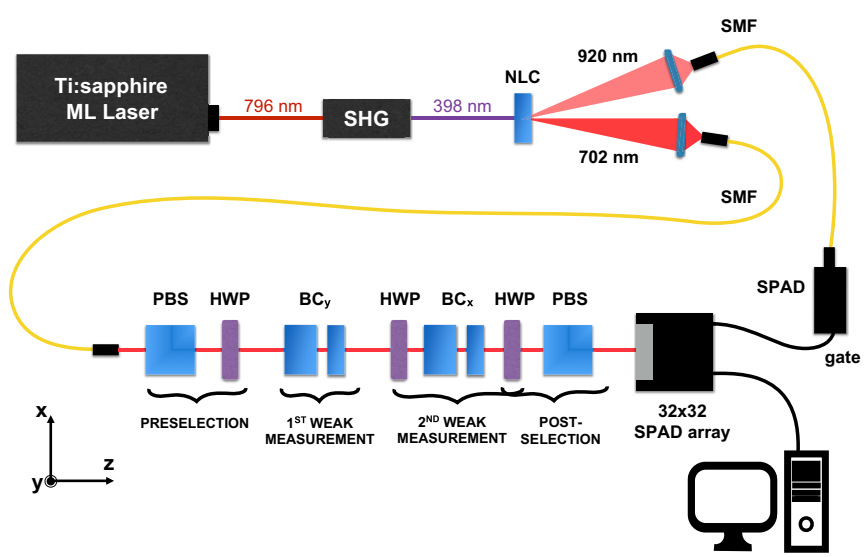

FIG. 1. Experimental setup. Heralded single photons are produced by downconversion in a 5 -mm-long $\mathrm{LiIO}_{3}$ nonlinear crystal (NLC) cut for type-I phase matching; the pump beam, obtained by second harmonic generation (SHG) of a mode-locked laser (rep. rate, $76 \mathrm{MHz})$, produces idler $\left(\lambda_{i}=920 \mathrm{~nm}\right)$ and signal $\left(\lambda_{s}=702 \mathrm{~nm}\right)$ photons, which are then coupled in a single-mode fiber (SMF). The idler photons are detected by means of a single-photon avalanche diode (SPAD), which imparts a trigger to the signal detection system. Signal photons are prepared in the polarization state $\left|\psi_{A}\right\rangle$ by means of a polarizing beam splitter (PBS) and a half-wave plate (HWP) $\left(I_{A}=1\right)$; then they pass through a birefringent system $B C_{y}$ that shifts them in the transverse $y$ direction, depending on their polarization, thus measuring $I_{C}$ weakly. $B C_{y}$ consists of two birefringent crystals: the first one realizes the weak interaction, while the second one compensates temporal walk-off and decoherence effects. A similar system, $B C_{x}$, performs in cascade a weak measurement of $I_{B}$ by shifting the photons in the $x$ direction; this is placed after a HWP allowing one to measure along an arbitrary linear polarization axis. A further HWP is used to counter the basis change and decide the observable $I_{D}$ which determines the postselection. The photons are finally detected by means of a spatial-resolving $32 \times 32$ SPAD array. 
Single photons are emitted by a downconversion source [59]; at a heralding rate around $130 \mathrm{kHz}$, the quality of the emission is certified by a measured value of the antibunching parameter [60] of $0.13 \pm 0.01$ without any background and dark-count subtraction. This implies that in our test we can genuinely associate the outcomes of the measurements to properties of single particles, avoiding classical wavelike analogies [61]. The state of the photon is prepared (preselected) in the polarization state $\left|\psi_{A}\right\rangle=\cos \alpha|H\rangle+\sin \alpha|V\rangle$ by means of a calcite polarizing beam splitter (PBS) and a half-wave plate (HWP).

The use of a single-mode fiber (SMF) then prepares the transverse profile $\mathcal{F}(x, y)$ in a Gaussian shape of width $\sigma$, which ensures that the two directions can be used as distinct pointers for the weak measurements [56]. These operations are implemented by coupling the polarization to the transverse position by means of the unitary transformations $\widehat{U}_{x}=\exp \left(-i g_{x} \widehat{I}_{B} \otimes \widehat{P}_{x}\right)$ and $\widehat{U}_{y}=\exp \left(-i g_{y} \widehat{I}_{C} \otimes \widehat{P}_{y}\right)$, where $\widehat{I}_{C}=|H\rangle\langle H|-| V\rangle\langle V|$ and $\widehat{I}_{B}=\left|\psi_{\gamma}\right\rangle\left\langle\psi_{\gamma}|-| \psi_{\gamma}^{\perp}\right\rangle\left\langle\psi_{\gamma}^{\perp}\right|$ are associated with an arbitrary direction for the linear polarization: $\left|\psi_{\gamma}\right\rangle=\cos \gamma|H\rangle+\sin \gamma|V\rangle$ and $\left|\psi_{\gamma}^{\perp}\right\rangle=\sin \gamma|H\rangle-$ $\cos \gamma|V\rangle$. The operators $\widehat{P}_{x}$ and $\widehat{P}_{y}$ are the momenta associated with the $x$ and $y$ positions, respectively. The interaction $\widehat{U}_{x}$ $\left(\widehat{U}_{y}\right)$ is brought about by a 2 -mm-long birefringent crystal whose extraordinary (e) optical axis lies in the $x-z(y-z)$ plane, at a $\pi / 4$ angle with respect to the $z$ direction. Due to the spatial walk-off effect experienced by the photons, the two polarization paths get slightly separated along the $x(y)$ direction. The actual interaction along the $I_{B}$ basis can be tuned by means of a HWP. The condition $g_{x}^{2} / \sigma^{2} \simeq$ $g_{y}^{2} / \sigma^{2} \ll 1$ ensures that the back-action on the incoming state is negligible; i.e., the measurement is carried out in the weak regime [56]. Along with the spatial walk-off, each birefringent crystal also induces a temporal walk-off and a possible polarization change, both to be compensated to avoid unwanted additional decoherence effects. We were able to do this by adding after each crystal a second birefringent crystal of properly chosen length $(1.1 \mathrm{~mm})$ with the optical axis along the $y(x)$ direction, each mounted on a piezo-controlled rotator with $100 \mu \mathrm{rad}$ nominal resolution, allowing us to cancel the temporal walk-off, avoiding unwanted circular components in the polarization state due to the previous interaction.

After the second weak interaction, the photons arrive at a HWP that undoes the preceding rotation and, at the same time, determines the projection of the state onto one of the postselected states $\left\langle\psi_{A}\right|,\left\langle\psi_{D}\right|=\cos \delta\langle H|+\sin \delta\langle V|$, or $\left\langle\psi_{D}^{\perp}\right|=\sin \delta\langle H|-\cos \delta\langle V|$, by means of a PBS.

At the end of the optical path, the single photon is detected by a spatial-resolving single-photon detector prototype, i.e., a two-dimensional array made of $32 \times 32$ "smart pixels" [62]each embedding a SPAD detector and its front-end electronics for counting and timing single photons-operating in parallel with a global shutter readout. The SPAD array is operated in gated mode, with each count by the SPAD on the heralding arm triggering a 6-ns detection window in each pixel of the array. At our heralding rate of $\sim 130 \mathrm{kHz}$, the dark-count rate of the array is drastically reduced by the low-duty cycle, improving the signal-to-noise ratio.
Since we are interested in the LGT as a tool for probing quantumness, we estimate each term in the inequality (7) separately in our setup. The chain of weak interactions and the space-resolved detector allow us to reconstruct the expectation values $\left\langle I_{B}\right\rangle$ and $\left\langle I_{C}\right\rangle$ by measuring the average $x$ and $y$ positions of the photons, respectively, when postselecting on the input state $\left\langle\psi_{A}\right|:\langle\widehat{x}\rangle \simeq g_{x}\left\langle\widehat{I}_{B}\right\rangle$ and $\langle\widehat{y}\rangle \simeq g_{y}\left\langle\widehat{I}_{C}\right\rangle$. The correlation of the $x$ and $y$ positions gives $\langle\hat{x} \hat{y}\rangle \simeq$ $\frac{g_{x} g_{y}}{2}\left(\left\langle\widehat{I}_{B} \widehat{I}_{C}\right\rangle+\left\langle\widehat{I}_{B}\right\rangle\left\langle\widehat{I}_{C}\right\rangle\right)$. By inverting these relations, it is possible to obtain the single and sequential values $\left\langle\widehat{I}_{C}\right\rangle,\left\langle\widehat{I}_{B}\right\rangle$, and $\left\langle\widehat{I}_{B} \widehat{I}_{C}\right\rangle$, estimated as weak averages. This resolves a major difficulty, in that by using standard "strong" measurements one would only have access to the symmetrized quantity $\frac{1}{2}\left\langle\psi_{A}\left|\widehat{I}_{B} \widehat{I}_{C}+\widehat{I}_{C} \widehat{I}_{B}\right| \psi_{A}\right\rangle$ [63]. Postselection on $\left\langle\psi_{D}\right|$ and $\left\langle\psi_{D}^{\perp}\right|$ occurrence delivers the probabilities $p_{D}(1)=\left|\left\langle\psi_{D} \mid \psi_{A}\right\rangle\right|^{2}$ and $p_{D}(-1)=\left|\left\langle\psi_{D}^{\perp} \mid \psi_{A}\right\rangle\right|^{2}$, as well as the weak values ${ }_{1}\left\langle\widehat{I}_{C}\right\rangle$ and ${ }_{-1}\left\langle\widehat{I}_{C}\right\rangle$. We are then asking the macrorealist to agree that this experimental procedure delivers the correct results because it accesses the actual "macrorealist values" of the observables, since postselection on the input state leads to the expected value, with no anomalies. In this respect, it is not surprising that the measurement procedure is insensitive to the imaginary part of the weak values [64], as discussed in Ref. [56].

\section{RESULTS AND CONCLUSIONS}

Figure 2 reports a theoretical simulation showing the shape of $\mathcal{B}_{4}$ for four different values of $\gamma$, plotted vs the parameters $\alpha$ and $\delta$ determining the pre- and postselection states. Aside from
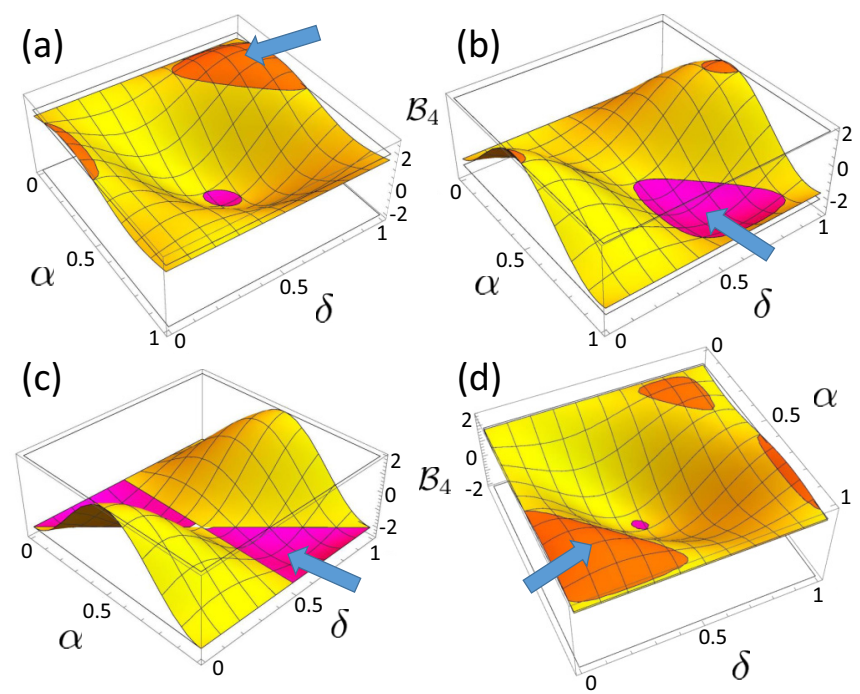

FIG. 2. Behavior of the quantity $\mathcal{B}_{4}$ in Eq. (7) vs the parameters $\alpha$ (related to the state $\left|\psi_{A}\right\rangle$ ) and $\delta$ (determining the states $\left|\psi_{D}\right\rangle$ and $\left|\psi_{D}^{\perp}\right\rangle$ ), both in $\pi$ units, for four different values of the parameter $\gamma$ defining the polarization operator $I_{B}: \gamma=0.1 \pi$ for plot (a), $\gamma=0.4 \pi$ for plot (b), $\gamma=0.5 \pi$ for plot (c), and $\gamma=0.95 \pi$ for plot (d). In each of these plots, the yellow part of the surface indicates the nonviolation area $\left(-2 \leqslant \mathcal{B}_{4} \leqslant 2\right)$, while in orange and magenta are highlighted respectively the positive $\left(\mathcal{B}_{4}>2\right)$ and negative $\left(\mathcal{B}_{4}<-2\right)$ violation areas. In each plot, the blue arrow indicates the point for which the violation was experimentally checked. 
TABLE I. Leggett-Garg inequality violation results obtained in our four experimental scenarios. The first column reports the $\gamma, \alpha$, and $\delta$ values exploited in each experiment, the second and third columns host, respectively, the theoretical $\left(\mathcal{B}_{4}^{\text {(th) }}\right)$ and experimentally obtained $\left(\mathcal{B}_{4}^{(\exp )}\right.$ ) values of the quantity $\mathcal{B}_{4}$, while the fourth and fifth columns show the anomalous weak values obtained for $I_{C}$ in each experiment.

\begin{tabular}{lcccc}
\hline \hline Parameters & $\mathcal{B}_{4}^{\text {(th) }}$ & $\mathcal{B}_{4}^{\text {(exp) }}$ & ${ }_{1}\left\langle I_{c}\right\rangle$ & ${ }_{1}\left\langle I_{c}\right\rangle$ \\
\hline$\gamma=0.1 \pi$ & & & \\
$\alpha=0.233 \pi$ & 2.82 & $2.76 \pm 0.17$ & $2.34 \pm 0.04$ & $-0.34 \pm 0.04$ \\
$\delta=0.867 \pi$ & & & \\
$\gamma=0.4 \pi$ & & & \\
$\alpha=0.767 \pi$ & -2.82 & $-2.74 \pm 0.18$ & $-0.30 \pm 0.04$ & $2.20 \pm 0.04$ \\
$\delta=0.633 \pi$ & & & \\
$\gamma=0.5 \pi$ & & & \\
$\alpha=0.833 \pi$ & -2.50 & $-2.56 \pm 0.16$ & $0.01 \pm 0.06$ & $1.86 \pm 0.06$ \\
$\delta=0.667 \pi$ & & & \\
$\gamma=0.95 \pi$ & & & \\
$\alpha=0.8 \pi$ & 2.71 & $2.86 \pm 0.19$ & $1.86 \pm 0.04$ & $-0.12 \pm 0.06$ \\
$\delta=0.15 \pi$ & & & & \\
\hline \hline
\end{tabular}

the yellow part of the surface, indicating where the classical bound holds, for each $\gamma$ value one observes orange and/or magenta areas, corresponding to the $\mathcal{B}_{4}>2$ and $\mathcal{B}_{4}<-2$ violations, respectively.

We tested the inequality for different choices of the initial state $\alpha$, of the orientation $\gamma$ of the weak measurement, and of the final postselection $\delta$ : the four combinations have been identified to deliver a violation (indicated by the blue arrow in each plot reported in Fig. 2) close to the maximal value, whose results are illustrated in Table I. For each of the four tests performed, the experimental values of $\mathcal{B}_{4}$ are in excellent agreement with the theoretical expectations within the statistical uncertainties, granting for both the positive and negative values a classical bound violation between 3.4 and 4.4 standard deviations. In Table I, we also report the measured weak values showing how anomalies, i.e., values outside the standard range -1 to 1 , do flag the violation of the Leggett-Garg inequality when these obey the condition (8).

We demonstrated the capability of our setup to address single photons with negligible disturbance, certifying it by a LGT and, in a complementary way, by the presence of anomalous weak values upon postselection. This is a manifestation of the good quality of our device, which may find applications in random number generators [65-67]. Care must be taken, however, in extending these considerations to adversarial scenarios, when the presence of loopholes, either from fair sampling or from "clumsiness" of the experimenter $[37,68]$, can play a role.

\section{ACKNOWLEDGMENTS}

This work has been supported by EMPIR-14IND05 "MIQC2" (the EMPIR initiative is cofunded by the EU H2020 and the EMPIR Participating States) and the MIUR Progetto Premiale 2015 "Q-SecGroundSpace”.

\section{APPENDIX}

We can generalize the connection between the violation of a LGI and postselected values as follows: consider a sequence of $m$ binary measurements $I_{n}$, with the first one $I_{1}$ coinciding with state preparation, hence $I_{1}=1$ deterministically. A generalized LGI is written as

$$
-n \delta_{n=2 k+1}-(n-2) \delta_{n=2 k} \leqslant \mathcal{B}_{n} \leqslant n-2,
$$

with

$$
\mathcal{B}_{n}=\sum_{m=1}^{n-2}\left\langle I_{m} I_{m+1}\right\rangle+\left\langle I_{n-1} I_{n}\right\rangle-\left\langle I_{1} I_{n}\right\rangle .
$$

We now consider the last measurement $I_{n}$ and distinguish between the events for which $I_{n}=1$ or $I_{n}=-1$, each occurring with probabilities $p_{+}$and $p_{-}$, respectively. This leads us to consider the postselected values ${ }_{ \pm}\left\langle I_{n} I_{n+1}\right\rangle$ for any correlator in (A1):

$$
\begin{aligned}
\mathcal{B}_{n}= & p_{+}\left(\sum_{m=1}^{n-2}{ }_{+}\left\langle I_{m} I_{m+1}\right\rangle+{ }_{+}\left\langle I_{n-1}\right\rangle-1\right) \\
& +p_{-}\left(\sum_{m=1}^{n-2}-\left\langle I_{m} I_{m+1}\right\rangle-{ }_{-}\left\langle I_{n-1}\right\rangle+1\right) .
\end{aligned}
$$

If we now assume that all postselected values are regular, in that they are both within the spectrum of ordinary eigenvalues, the term in $p_{+}$is upper bounded by $n-2$. The term in $p_{-}$actually contains an expression akin to $\mathcal{B}_{n-1}$ for the postselected values; the upper bound for the whole quantity is $n-2$ as well. Therefore, the regularity of the postselected values in both their domain and their compatibility with macroscopic realism, leads to the LGI (A1).
[1] M. Genovese, Adv. Sci. Lett. 3, 249 (2010).

[2] J. S. Bell, Speakable and Unspeakable in Quantum Mechanics (Cambridge University, Cambridge, England, 1987).

[3] M. Genovese, Phys. Rep. 413, 319 (2005), and references therein.

[4] S. Kochen and E. P. Specker, J. Math. Mech. 17, 59 (1968).

[5] A. J. Leggett and A. Garg, Phys. Rev. Lett. 54, 857 (1985).

[6] J. Kofler and Č. Brukner, Phys. Rev. Lett. 99, 180403 (2007).

[7] J. Kofler and Č. Brukner, Phys. Rev. Lett. 101, 090403 (2008).

[8] M. Barbieri, Phys. Rev. A 80, 034102 (2009).
[9] N. Lambert, C. Emary, Y.-N. Chen, and F. Nori, Phys. Rev. Lett. 105, 176801 (2010).

[10] S. Nimmrichter and K. Hornberger, Phys. Rev. Lett. 110, 160403 (2013).

[11] C. Budroni and C. Emary, Phys. Rev. Lett. 113, 050401 (2014).

[12] C. Robens, W. Alt, D. Meschede, C. Emary, and A. Alberti, Phys. Rev. X 5, 011003 (2015).

[13] C. Budroni, G. Vitagliano, G. Colangelo, R. J. Sewell, O. Gühne, G. Tóth, and M. W. Mitchell, Phys. Rev. Lett. 115, 200403 (2015). 
[14] P. Facchi et al., J. Phys. A 47, 035301 (2014).

[15] A. Palacios-Laloy, F. Mallet, F. Nguyen, P. Bertet, D. Vion, D. Esteve, and A. N. Korotkov, Nat. Phys. 6, 442 (2010).

[16] M. E. Goggin, M. P. Almeida, M. Barbieri, B. P. Lanyon, J. L. O'Brien, A. G. White, and G. J. Pryde, Proc. Natl. Acad. Sci. USA 108, 1256 (2011).

[17] J. Dressel, C. J. Broadbent, J. C. Howell, and A. N. Jordan, Phys. Rev. Lett. 106, 040402 (2011).

[18] V. Athalye, S. S. Roy, and T. S. Mahesh, Phys. Rev. Lett. 107, 130402 (2011).

[19] G. C. Knee, S. Simmons, E. M. Gauger, J. J. Morton, H. Riemann, N. V. Abrosimov, P. Becker, H.-J. Pohl, K. M. Itoh, M. L. Thewalt, G. A. D. Briggs, and S. C. Benjamin, Nat. Commun. 3, 606 (2012).

[20] Y. Suzuki, M. Iinuma, and H. F. Hofmann, New J. Phys. 14, 103022 (2012).

[21] Z.-Q. Zhou, S. F. Huelga, C.-F. Li, and G.-C. Guo, Phys. Rev. Lett. 115, 113002 (2015).

[22] G. C. Knee, K. Kakuyanagi, M.-C. Yeh, Y. Matsuzaki, H. Toida, H. Yamaguchi, S. Saito, A. J. Leggett, and W. J. Munro, Nat. Commun. 7, 13253 (2016).

[23] G. Brida, I. P. Degiovanni, M. Genovese, F. Piacentini, V. Schettini, N. Gisin, S. V. Polyakov, and A. Migdall, Phys. Rev. A 79, 044102 (2009).

[24] C. Emary, N. Lambert, and F. Nori, Rep. Prog. Phys. 77, 016001 (2014), offers a comprehensive review of recent results.

[25] Č. Brukner, S. Taylor, S. Cheung, and V. Vedral, arXiv:quant-ph/0402127.

[26] T. Fritz, New J. Phys. 12, 083055 (2010).

[27] A. Fedrizzi, M. P. Almeida, M. A. Broome, A. G. White, and M. Barbieri, Phys. Rev. Lett. 106, 200402 (2011).

[28] C. Budroni, T. Moroder, M. Kleinmann, and O. Gühne, Phys. Rev. Lett. 111, 020403 (2013).

[29] S. Brierley, A. Kosowski, M. Markiewicz, T. Paterek, and A. Przysiezna, Phys. Rev. Lett. 115, 120404 (2015).

[30] C. Marletto and V. Vedral, npj Quantum Inf. 3, 41 (2017).

[31] J.-S. Tang et al., Chin. Phys. Lett. 28, 060304 (2011).

[32] Y. Aharonov and L. Vaidman, Phys. Rev. A 41, 11 (1990).

[33] H. M. Wiseman, Phys. Rev. A 65, 032111 (2002).

[34] A. N. Jordan, A. N. Korotkov, and M. Buttiker, Phys. Rev. Lett. 97, 026805 (2006).

[35] N. S. Williams and A. N. Jordan, Phys. Rev. Lett. 100, 026804 (2008).

[36] J. P. Groen, D. Ristè, L. Tornberg, J. Cramer, P. C. de Groot, T. Picot, G. Johansson, and L. DiCarlo, Phys. Rev. Lett. 111, 090506 (2013).

[37] T. C. White et al., npj Quantum Inf. 2, 15022 (2016).

[38] C. Ferrie and J. Combes, Phys. Rev. Lett. 113, 120404 (2014).

[39] A. Brodutch, Phys. Rev. Lett. 114, 118901 (2015).

[40] C. Ferrie and J. Combes, Phys. Rev. Lett. 114, 118902 (2015).
[41] Y. Aharonov and D. Rohrlich, arXiv:1410.0381.

[42] L. Vaidman, arXiv:1409.5386 (2014).

[43] L. Vaidman, arXiv:1402.0199 (2014).

[44] Y. Aharonov, D. Z. Albert, and L. Vaidman, Phys. Rev. Lett. 60, 1351 (1988).

[45] G. J. Pryde, J. L. O’Brien, A. G. White, T. C. Ralph, and H. M. Wiseman, Phys. Rev. Lett. 94, 220405 (2005).

[46] N. S. Williams and A. N. Jordan, Phys. Rev. Lett. 103, 089902(E) (2009).

[47] M. Pusey, Phys. Rev. Lett. 113, 200401 (2014).

[48] F. Piacentini, A. Avella, M. P. Levi, R. Lussana, F. Villa, A. Tosi, F. Zappa, M. Gramegna, G. Brida, I. P. Degiovanni, and M. Genovese, Phys. Rev. Lett. 116, 180401 (2016).

[49] O. Hosten and P. G. Kwiat, Science 319, 787 (2008).

[50] P. B. Dixon, D. J. Starling, A. N. Jordan, and J. C. Howell, Phys. Rev. Lett. 102, 173601 (2009).

[51] A. Feizpour, X. Xing, and A. M. Steinberg, Phys. Rev. Lett. 107, 133603 (2011).

[52] A. N. Jordan, J. Martínez-Rincón, and J. C. Howell, Phys. Rev. X 4, 011031 (2014).

[53] S. Pang and T. A. Brun, Phys. Rev. Lett. 115, 120401 (2015).

[54] Y. Turek, W. Maimaiti, Y. Shikano, C.-P. Sun, and M. Al-Amri, Phys. Rev. A 92, 022109 (2015).

[55] L. Zhang, A. Datta, and I. A. Walmsley, Phys. Rev. Lett. 114, 210801 (2015).

[56] F. Piacentini, A. Avella, M. P. Levi, M. Gramegna, G. Brida, I. P. Degiovanni, E. Cohen, R. Lussana, F. Villa, A. Tosi, F. Zappa, and M. Genovese, Phys. Rev. Lett. 117, 170402 (2016).

[57] J. F. Clauser, R. A. Holt, A. Shimony, and M. A. Horne, Phys. Rev. Lett. 23, 880 (1969).

[58] J. Dressel and A. N. Korotkov, Phys. Rev. A 89, 012125 (2014).

[59] S. Castelletto, I. P. Degiovanni, V. Schettini, and A. Migdall, Opt. Express 13, 6709 (2005).

[60] P. Grangier, G. Roger, and A. Aspect, Eur. Phys. Lett. 1, 173 (1986).

[61] J.-S. Xu, C.-F. Li, X.-B. Zou, and G.-C. Guo, Sci. Rep. 1, 101 (2011).

[62] F. Villa, R. Lussana, D. Bronzi, S. Tisa, A. Tosi, F. Zappa, A. Dalla Mora, D. Contini, D. Durini, S. Weyers, and W. Brockherde, IEEE J. Sel. Top. Quantum Electron. 20, 3804810 (2014).

[63] G. Mitchison, R. Jozsa, and S. Popescu, Phys. Rev. A 76, 062105 (2007).

[64] R. Jozsa, Phys. Rev. A 76, 044103 (2007).

[65] F. J. Curchod, M. Johansson, R. Augusiak, M. J. Hoban, P. Wittek, and A. Acin, Phys. Rev. A 95, 020102(R) (2017).

[66] M. Schiavon, L. Calderaro, M. Pittaluga, G. Vallone, and P. Villoresi, Quantum Sci. Technol. 2, 015010 (2017).

[67] M. J. Hu et al., arXiv:1609.01863.

[68] M. M. Wilde and A. Mizel, Found. Phys. 42, 256 (2012). 